vitreous. If the amount effused be small, although the absorption of it is, as a rule, very slow, yet after a few weeks or months it becomes altered in character ; it loses its colouring matter, and the clot shrinks; and it is seen, with the aid of ophthalmoscopic light, as a small dark mass, or it may be as filamentous shreddy particles in the vitreous, and in this condition it may remain for years. When the quantity effused into the vitreous is large, loss of the eye is almost certain to follow; not necessarily an immediate loss, but the chronic secondary changes which are the result of it only end in the destruction of all visual power. To allow the blood to be extravasated into the vitreous, the hyaloid has to be ruptured; and wherever the blood forces its way, it breaks down the texture of the vitreous. If, therefore, a large extent of the vitreous space be occupied by blood, the whole vitreous as a consequence suffers. From impairment of its nutrition, it atrophies, loses its consistence, and becomes fluid. In these large effusions of blood into the vitreous, although the clot may remain for a few weeks or months unabsorbed, yet with the change which takes place in the vitreous, it is slowly disposed of. As the vitreous becomes fluid, the clot softens and gradually disappears; but it stains the whole of the fluid which occupies the vitreous space to a yellow or brownish-yellow tinge, which colour may last for years. The mischief, however, does not end here; for, as the vitreous becomes fluid and the clot is absorbed, it diminishes in bulk; and the retina, losing the support which it had received from the healthy vitreous, falls forward and becomes detached.

Wound in the Sclerotic close to the Margin of the Cornea, from a Fragment of Iron detached from a Rivet; Large Prolapse of the Iris; Homorrhage into the Anterior Chamber, and into the Vitreous, from Injury to the Ciliary Processes; Excision of the Eye three months after the Accident. William R., aged 53, a boiler-maker, came to the hospital on September 26th, 1864, suffering from an injury he had received the day previously. Whilst cutting a cold rivet, a sharp-edged fragment of it flew off and struck the left eye, inflicting a wound in the sclerotic nearly a quarter of an inch in length, close to the lower and outer margin of the cornea.

State on Admission. The anterior chamber was full of blood, so as to prevent any of the deeper parts being seen. Through the wound above indicated, there was an extensive prolapse of the iris, which was jagged and torn. It was also probable that vitreous had been lost. The protruded lacerated portion of iris was removed with a pair of scissors; a cottonwool compress was applied to the eye, and two leeches to the temple, to be repeated if necessary; and a belladonna lotion was ordered to bathe the eye with three or four times a day.

September 30th. A large part of the blood in the anterior chamber had been absorbed; and it was now evident that nearly the whole of the lens had escaped through the wound, and that there had been extensive hæmorrhage into the vitreous. A small quantity of opaque lenticular matter still remained; but, behind this, a large clot of blood could be seen in the anterior part of the vitreous space, immediately behind the ciliary processes from which the hæmorrhage had probably ensued. The eye was much inflamed, with chemosis of the conjunctiva, and very painful. The leeches to the temple were repeated. From this date, all active inflammation gradually subsided; and in about six weeks he was able to leave the hospital. All sight was lost. The blood in the anterior chamber was completely absorbed; but the clot in the vitreous still remained, though diminished in size.
In January of 1865 , he was re-admitted into the hospital, as the lost eye had become inflamed and was giving him pain. It was quite blind, very soft, and shrinking; being already smaller than the sound eye. The blood in the vitreous had quite disappeared, and the retina was detached. Under these circumstances, I advised him to have the eye removed; and he having consented, I excised it. On making a section of it, the following was the condition. The lower and outer third of the iris was gone. There was no lens; but an opaque film remained, which was united to the posterior surface of the iris. There was no vitreous; but the retina was entirely detached and coarcted, fastened posteriorly around the optic nerve, and anteriorly adherent to the back of the iris. The space between the retina and choroid was filled with a yellow serous fluid. The choroid was in situ throughout, and in contact with the sclerotic except at several points where small ecchymoses separated them.

In this patient, it is probable that, in addition to the extensive hæmorrhage into the vitreous from the ciliary processes, there was also some hæmorrhage between the choroid and retina; but the blood in the anterior part of the eye prevented the fundus from being seen. The falling forward and complete detachment of the retina, is however a result which is often met with after a portion of the vitreous has been lost through a wound of the eye, and the remainder of it has been broken down by an extravasation of blood within it.

5, Harley Street, Cavendish Square.

\section{A CASE OF THORACIC ANEURISM SUCCESSFULLY TREATED BY REST.}

By A. T. H. Waters, M.D., Physician to the Liverpool Northern Hospital.

THE interest attaching to all cases of thoracic aneurism, and to any measures which afford a prospect of relief in that disease, induce me to lay the following case, although an isolated one, under the notice of the profession, in hope that the practice which has, in this instance, been attended with very satis. factory results, may have a wider trial than has hitherto been given to it.

From the opportunities I have had of treating cases of thoracic aneurism, I am led to believe that but little good can be done by the administration of any drug. I have in several cases of late years given a fair trial to various modes of treatment; and, although I have seen improvement sometimes follow admission into hospital, from the rest, comparative quiet, and other treatment to which the patients have been subjected, I have never seen such marked benefit follow as in the case below.

The treatment of internal aneurism by absolute rest has lately been strongly advocated by Mr. Tufnell of Dublin; and he has recorded some very interesting cases, in which he adopted the practice successfully. I have carried out in my case the line of practice he has recommended, which consists essentially in keeping the patient in the recumbent posture, and in administering a restricted diet. The results of the case are, I think, the more satisfactory, inasmuch as various other modes of treatment-moderate diet-iodide of potassium-ice to the tumour - acetate of lead-had been tried without any success, before the treatment by rest was commenced.

The well known effect produced on the circulation by the recumbent posture was well illustrated in my patient; the pulse having diminished by from ten to 
twenty beats per minute. To this circumstance the favourable issue must, I think, be mainly attributed.

CAse. John L., a married man, 45 years of age, was admitted into the Liverpool Northern Hospital, under my care, on December 14th, 1864. He applied to the hospital in consequence of a pain in his back; but, on examination, a pulsating tumour was found below the right clavicle, He gave the following history.

For the last seventeen years he had been a shipkeeper on night duty, but before that time he worked hard as a labourer. He had been accustomed to drink freely. About seventeen years ago, his right arm was amputated for disease of the elbow-joint following an accident. About three years ago he had a fall, by which he broke some of the ribs of his right side. For several years he had had rheumatic pains in the limbs and stump. For twelve months before admission, he had had violent attacks of sickness and cough; but he had never expectorated any blood. About seven or eight months before admission, he noticed a pain of a shooting character just below the right clavicle; and, about four months before admis. sion, he felt a pulsating lump in the same spot. This lump, he said, had grown very slowly, and was scarcely larger than when he first felt it. $\mathrm{He}$ seemed to attach but little importance to it, and considered the pain in the back his chief ailment. On one occasion, whilst walking in the strects of Liverpool in September 1864, he had what he described as a fit, which appears to have been of a syncopal nature.

Physical Condition, etc. Below the right clavicle, opposite the lower margin of the second rib, a little external to its junction with its cartilage, there was a tumour, conical in form, with a rounded apex. The tumour had pushed the rib outwards, and appeared immediately beneath the skin. The visible portion was about an inch and a half in diameter. It had a soft fluctuating feel, as if containing fluid. It was the seat of pulsation, which was visible, expansile, heaving, and of moderate force. Pressure diminished the size of the tumour. Percussion revealed an area of dulness, bounded above by the clavicle, below by the third rib, internally by the median line of the sternum, and externally reaching nearly to the nipple-line. 'There was normal resonance on the left side of the chest. Over the tumour, and below the middle of the right clavicle, a loud murmur was heard synchronous with the systole of the heart. A soft systolic murmur was heard at the apex of the heart. The pulse was small, regular, of equal size on both sides. The patient was somewhat emaciated, had sharp features, and an anxious expression of countenance. He complained of severe pain along the back; and there was some tenderness at its lower part. 'There was no pain over the tumour. He had a severe barking cough, and dyspncea on exertion; but there was no dysphagia. The veins of the left arm were rather large and knotted, but there was no cedema of the arm, nor of the stump. The digestive organs were unaffected. The pupils of both eyes were of the same size.

The patient, on admission, was put on a moderately spare diet, a small quantity of meat being allowed daily. The first remedy that was tried was iodide of potassium, which was given in large and gradually increasing doses, until the quantity given was twenty grains three times a day. This treatment was continued for some time. No perceptible effect was produced on the aneurism; but the patient's health began to suffer, and the remedy was accordingly stopped.

After an interval of some weeks, the application of ice was tried. The patient was kept in bed, and a bag of ice was applied over the tumour during the day. This was continued for many days; but no noticeable change resulted in the aneurism.

On March 21st, three grains of acetate of lead, 흠 with half a grain of opium, were ordered to be taken $\overline{\bar{\omega}}$ three times a day. This treatment was continued for a week, when symptoms came on which induced me to discontinue it.

On April 6th, in consequence of a good deal of pain in the chest being complained of, six ounces of blood were taken away by venesection. This was followed by a diminution of the pain.

On April 18th, the patient was ordered to confine himself entirely to his bed, and to keep in the horizontal posture. He had, up to this time, been in the hospital more than four months; and no perceptible change for the better or worse had, as far as could be observed, occurred. He was put on a very restricted diet; no stimulants were given, and, throughout the treatment, no medicines except an occasional purgative or anodyne. These, however, were but rarely required. The man was intelligent, and tolerably manageable; and, with one exception, when be walked from one ward to another during the time when the hospital was being cleaned, I believe he scarcely moved from the horizontal posture for a period of nearly eleven weeks. During this time, his health continued good; he slept well; complained of but little pain; had no sickness; and his cough became less frequent. His pulse (which, before the treatment was commenced, used to average from 80 to 90 ) fell to from 60 to 70 . On one occasion, a few days before he was kept in bed, it was found as follows: Standing, 92 ; sitting, 84; lying, 70 per minute.

The diet which was ordered for the patient was, seven ounces of bread, three ounces of meat, and eight ounces of fiuid daily. He was allowed small quantities of ice to relieve thirst, and to smoke one pipe daily. For some weeks after the treatment was commenced, more fluid than was ordered was taken, probably about a pint daily, but subsequently the above quantity (eight ounces) was rigidly adhered to.

Towards the end of May, it was very evident, from the diminished elasticity and pulsation in the tumour, that consolidation of the sac was taking place; and towards the middle of June there could be ro doubt that this result had been produced. The patient was allowed to get up during the twelfth week of treatment. He was, however, kept in the hospital till August 12th, when he was discharged.

The following are the notes taken of the condition of the aneurism, etc., at the termination of the treatment.

"The tumour has diminished in prominence, in size, and in area of dulness. Pulsation is felt over the whole tumour; it is distant, and gives the sensation as if a good deal of solid substance existed between the skin and the inside of the tumour. A systolic murmur is faintly heard over the tumour, and at the apex a double knock is felt. In the $\downarrow$ pex or presenting portion, a very remarkable change has taken place: instead of giving the sensation, as at first, of a soft fluctuating swelling very like that of an abscess, it is now hard, and feels like a solid mass. The pulsation at this spot, where it was originally most marked, is now least so, and can only be discovered on careful examination."

I have seen the patient repeatedly since his discharge from the hospital. He is able to go about, and fieels quite equal to undertake his former work of ship-watcher. He scarcely complains of the pulsation of his aneurism. I have quite recently examined his chest, and I found that the only per- 
ceptible changes that had occurred since his dismissal from the hospital were diminished prominence and pulsation of the tumour, with other indications of increased solidity.

\section{Cramsactions of 迶rametres.}

SOUTH MIDLAND BRANCH.

\author{
A CASE OF ANIMAL POISONING.
}

By William Newman, M.D.Lond., Stamford.

[Read October 26th, 1865.]

THE sources from which animal poisons may be supplied to the human frame are sufficiently numerous; with one, at least-the dissection-wound, or the post mortem puncture-medical men are far too familiar in their own persons.

I do not wish to occupy the time of the members of this Branch by a review of the literature of the subject, or by futile speculations on the materies mali, which may be transmitted from one member of the animal kingdom to another; but $I$ am anxious to bring before jou the particulars of a case which some short time back came under my notice, not because I have, or think that I have, any novelty in treatment to suggest, but merely that in calling attention to a serious form of disease, I may elicit from those present an expression of opinion as to the best mode of dealing with such cases, or awaken attention to our present inadequate knowledge. My case is as follows.

S. F., a veterinary surgeon, was called to some cattle ill near Uppingham, and saw them several times through the last week of July, and for the last time on August 2nd. The disease, supposed by himself to be the cattle-plague-more probably some form of blood-poisoning-had originated with some bullocks. These all, or nearly all, died. Several pigs were also attacked and died; and lastly a horse was affected. The horse, it would seem, had obtained access to a drinking-trough, the sides of which were smeared with puriform discharge from the nostrils of the diseased cattle. Two days afterwards, the horse was noticed to be ill, and within thirty-six hours from the first onset of symptoms was dead. It passed no urine from the time when it was first noticed to be ill.

S. F. made a post mortem examination of the horse, and gave me this verbal account of the appearances.

"The neck externally was very much swollen, and covered with abundant blisters holding a deep yellow serum. The deeper tissues were throughout infiltrated with the same kind of serous fluid, and the cervical glands were also much swollen. The lungs and kidneys were swollen und injected, and had local pus-deposits throughout their substance. The animals during life were covered with flies, and the carcases after death were equally infested."

At the time of making the post mortem examination, S. F. was in good health. He had had a few weeks before an attack of gout; this had passed by. He was a man who had lived freely; but there does not seem to have been reason for supposing that he was of actually intemperate habits. He had not, to his knowledge, any scratch or prick about his hand or arm; but, working with bare arms, and with his hands, etc., soaked in the fluids of the animal, he noticed that he was constantly covered with flies. From this time onwards he was not exposed to the influence of any animal virus, nor had he any further post mortem examination to undertake.

No symptoms showed themselves until August 9th (one week after the dissection of the horse). In the evening of that day, he felt his arm uneasy and noticed two little punctures-hardly visible without using a lens-on the left forearm at the junction of the lower and middle thirds, and toward the radial side of the back of the forearm. To these punctures he applied some nitrate of silver freely, making a black eschar fully the size of a crown-piece. In the course of the evening, he had creeping and general shivering more than once.

In the evening, Mr. Morgan, to whom I am much indebted for all preliminary detail and for the opportunity of seeing the patient, was called in, and prescribed some aperient.

Aug. 10th. The arm was more uneasy; an erysipelatous blush with some minute vesicles existed round the black eschar. The bowels were open.

Aug. 11th. The arm was more swollen; hand œdematous. Patches of very minute vesicles were scattered about the forearm. The swelling reach $\rightarrow d$ up to and slightly above the elbow. There was not the least sense of fluctuation. Through the twentyfour hours only about three ounces of urine were secreted.

Aug. 12th. The patient's appetite was failing; and he was worse in his general condition-uneasy and anxious about himself. The bowels were open; there was almost no urine; the conditions of the arm much the same.

Aug. 13th. I saw him with Mr. Morgan. His countenance was heavy and oppressed; he looked ill. Pulse 120, small. The skin was covered with abundant warm perspiration. His breathing was from 25-30 per minute. $\mathrm{He}$ had no headache: was restless at times. Only two or three ounces of urine were passed in the twenty-four hours; the bladder was empty. There was a blackened patch on the left arm, partly sore, partly covered with caustic eschar, discharging somewhat freely serous fluid, situated rather on the radial side as noted above. Ten or twelve large vesicles surrounded the central patch, and numerous smaller ones were scattered on the arm. The skin was firm and resisting, and there was not any sense, distinct or indistinct, of fluctuation. The arm was swollen considerably as far as the elbow; the back of the hand was puffy; the whole forearm was tender when touched, but, when let alone, was simply heary - not the seat of absolute pain. 'The whole conditions were very much those of well-marked phlegmasia dolens, with superadded vesicular affection and skin redness. The axillary glands were not in any degree affected. He was ordered to take a drachm of sulphite of soda every four hours. He took one or two doses of the soda; then sickness came on, and he was not able to continue the remedy.

Aug. 14th, 9 A.m. He was worse; said he must die. He was free from pain; had had some sleep. There was almost constant sickness. His intellect was clear. The vesicles were full of deep yellow serum. The arm was much as on the previous day in point of size and appearance.

9 P.M. I saw him again with Mr. Morgan. He was anxious and restless. The skin was perspiring freely ; pulse 120, very small; respiration at least 36 . He had no cough nor expectoration. He was sick very frequently; retched often, but brought up only a little mucus beyond the food taken. Little over an ounce of urine had been passed through the day. The arm was much more swollen both above and below the elbow; it was covered all over with resications, some very large and full of bloody fluid. The hand was swollen, puffy, and cold, and the arteries could not be felt. 'Three free incisions were made; one above the elbow, one near the eschar towards 